\title{
Analysis and Calculation of Horizontal Well in Heavy Oil Reservoir with Multipoint Injection Pipe String in Fluid Flow Pressure
}

\author{
$\mathrm{Xu} \mathrm{Xin}^{1,2}$ \\ ${ }^{1}$ Key Laboratory of enhanced oil recovery, Ministry of education, Northeast Petroleum \\ University ${ }^{2}$ Liaohe Oil Field Company, CNPC
}

Keywords: horizontal well; multipoint steam injection; pressure; calculation

\begin{abstract}
With the progress of the technical level of oil development, the application of horizontal wells is becoming more and more common. It is the key to improve the overall movement of the reservoir by using the horizontal well. The multipoint steam injection string of the horizontal well is a commonly used downhole component for high efficiency steam injection of the thermal recovery horizontal well. A reasonable layout of the valves to ensure an even steam output of each point is still not well designed. At present, a general design method which is urgently necessary isn't applied by each factory. According to the balance relationship of the momentum, the energy and the mass of the fluid flowing in the string, the pressure distribution equation of the fluid flowing in the multipoint steam injection string is built. On the basis of this equation, the design calculation method of the multipoint steam injection string is come up with. A specific example is given in the paper. Study shows that, with the equation built in this paper, the string can be designed with each axial valve having the same output, so that the technical demand of an even steam injection of the horizontal well section can be met.
\end{abstract}

\section{Introduction}

Multipoint steam injection of the horizontal well refers to a number of steam injection valves (or distributing pup joint) are setted at some spacing on the axial section of the long horizontal steam injection string, when steam is injected, each steam injection valve act on the horizontal section at the same time to achieve even steam inhaust by horizontal section, finally, solve the problem of uneven oil developed in the reservoir along the horizontal well. The author thinks that, the most important technical parameters should be the inside and outside pressure distribution of the horizontal interval.

At present, most of the steam injection string and its accessories used by oil field are proprietary product ${ }^{[3 \sim 5]}$. Producers can offer products according to the production, but can't give the design detail. The determination methods of the important parameters are regarded as trade secrets by the relevant manufacturers. And, furthermore, the design standards of the string structure used by every oil region are not unified and normative. This situation has restricted the perfection of high efficiency steam injection technology of the horizontal well, needs to be amended. Aiming at the 
above problems caming across the production of oil field, the fundamental research of the steam injection string design according to the hydromechanics theory is carried out. And the flow pressure distribution regularities in the multipoint steam injection string of a horizontal well is Analysised. All this work is aimng to providing a method of analysis and calculation for the steam injection string design of the horizontal well.

\section{Basic Assumptions and Basic Equations}

\subsection{Momentum Balance Equation}

The establishment of momentum balance equation is based on a control sample caming from axial steam injection string, as shown in figure 1 (a). The friction on pipe wall is assumed to be negligible. Also, it is assumed that the flow direction of effluence out of side holes is perpendicular to that of the fluid inside string. It means that the flow of side holes have no axial momentum. So, the momentum equation of the axial flow in horizontal string can be written as the equation below:

$$
P_{2}-P_{1}=\rho\left(u_{1}^{2}-u_{2}^{2}\right)
$$

A decrease in the flow quantity inside string is due to the diffluence by the side holes on the string. The section areas of cross-section 1 and 2 are same. So, $u_{2}<u_{1}, P_{2}>P_{1}$. Therefore, the pressure in the string will rises along with the fluid flow through the holes. It is known as the pressure recovery.

Due to the flow direction of effluence out of side holes isn't perpendicular to that of the fluid inside string strictly, the axial momentum won't disappears completely. So, there should be a term considered of this momentum at the rightside of equality in the equation (1). Because this term is ignored in the equation (1), so, the pressure recovery provided by equation (1) will be larger.

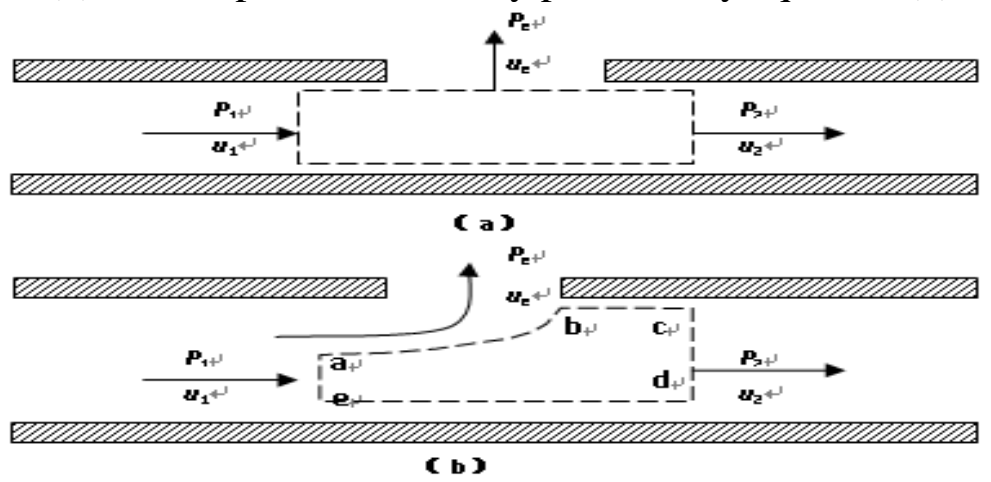

Fig.1 analytical diagram of momentum balance and energy balance

\subsection{Energy Balance Equation}

The flow fluid can be divided into two parts as shown in Fig.1 (b). Part of the fluid flow through holes at the flow velocity of ue, and the others continue to flow down in the string. It is assumed that the fluid flowing downstream come from the ae portion of figure completely. It means that downstream fluid doesn't flow through the ab. Obviously, this is an ideal situation. For section ae and cd, bernoulli's equation can be obtained:

$$
P_{2}-P_{1}=\frac{\rho}{2}\left(u_{1}^{2}-u_{2}^{2}\right)
$$

It must be pointed out that the results given by Eq. 1 and Eq. 2 are very rough. But both of them 
show that The value of the pressure is proportional to difference of two squares of the speed on the both sides of holes.

The comparison of Eq. 1 and Eq. 2 shows that the rise of pressure computed by the energy balance equation is only half of that getted through the momentum balance equation. Now correction coefficient $\mathrm{K}$ is introduced. The Eq. 1 and Eq. 2 can transform into the equation below:

$$
P_{2}-P_{1}=K \rho\left(u_{1}^{2}-u_{2}^{2}\right)
$$

$\mathrm{K}$ can be determined by experiment. $\mathrm{K}$ is always between 0 and 1 . Eq. 3 shows that, when the fluid is divided into two shares, the actual situation is between two situations which are indicated by conservation of mechanical energy and conservation of momentum.

\subsection{Mass Balance Equation}

It is assumed that the diameter of steam injection string is $\mathrm{D}$, and the diameter of the side holes is d. From the continuity equation of the incompressible fluid:

$$
u_{1}=u_{2}+\frac{d^{2}}{D^{2}} u_{e}
$$

The side holes of steam injection string is assumed to be circular, and the edges is assumed to be sharp. Fluid flow out to the sapce through the holes. The pressure of the space is $\mathrm{P}_{\mathrm{e}}$. Refer to Fig. 1 , according to the hydraulics theory, the calculation formula of $\mathrm{u}_{\mathrm{e}}$ can be written as:

$$
u_{e}=C \sqrt{\frac{2}{\rho}\left(\frac{P_{1}+P_{2}}{2}-P_{e}\right)}
$$

\section{Pressure Distribution Equation}

Eq. 3, Eq. 4 and Eq. 5 are established. Three variables $P_{2} 、 u_{2}$ and $u_{\mathrm{e}}$ are worked out through $P_{1}$, $u_{1}$ and $P_{\mathrm{e}} . P_{1}$ is upstream pressure of the side holes. $u_{1}$ is the speed of the fluid in the steam injection string. $P_{\mathrm{e}}$ is the exit pressure of the side holes.

Now, dimensionless parameter $a$ is defined. The expression of $a$ is below:

$$
a=\frac{K C d^{2}}{D^{2}}
$$

After proper operation, the equation of the flow pressure in the multipoint steam injection string of a horizontal well can be got as below:

yields

$$
P_{2}=P_{e}+\frac{K-a^{2}}{K+a^{2}}\left(P_{1}-P_{e}\right)+\frac{2 a^{2} K^{2}}{\left(K+a^{2}\right)^{2}} \rho u_{1}^{2}\left[1 \pm \sqrt{1+\frac{2\left(K+a^{2}\right)}{\left(K a^{2}\right) \rho u_{1}^{2}}\left(P_{1}-P_{e}\right)}\right]
$$

\section{Stress Calculation and Analysis of Examples}

By the examples, stress calculation method can be illustrated, and the sensitivity parameters which impact on the pressure distribution also can be analyzed.

Example: the closed steam injection string contains four side holes. The diameter of string $\mathrm{D}=$ $110 \mathrm{~mm}$; The diameter of the hole $\mathrm{d}=10 \mathrm{~mm}$; The distance between holes and it from hole to both ends is $2 \mathrm{~m}$; the intake velocity of fluid $\mathrm{u}=12.3 \mathrm{~m} / \mathrm{s}$, The outside pressure Pe is atmospheric 
pressure, $\mathrm{P}_{\mathrm{e}}=105$ pa. It is assumed that $\rho=1.185 \mathrm{~kg} / \mathrm{m} 3, \mu=1.842 * 10-5 \mathrm{~Pa} \cdot \mathrm{s}, \mathrm{K}=0.45, \mathrm{C}=0.62$. Try to solve the flow of each hole.

\subsection{Calculation method of interpretation}

The pressure drop from import to the first hole can be calculated by the formula of straight pipe pressure drop in hydraulics. The lateral flow of first hole ue, the the downstream velocity $\mathrm{u}_{2}$ and downstream pressure $\mathrm{P}_{2}$ of the hole can be got by Eq. 5, Eq. 4 and Eq. 7. The pressure drop between the first hole and the second hole can also be calculated by formula of straight pipe pressure drop in hydraulics. Then, The lateral flow of second hole, the the downstream velocity and downstream pressure of this hole are calculated. And this step is repeated until the last hole.

If the string is Endcapped, the downstream velocity of the last hole is zero. So, it requires that the determination of string inlet pressure must satisfy this condition. Therefore, the determination of inlet pressure requires iterative calculation.

If the fluid in the string is gas (vapor)-liquid two-phase fluid, the monophasic flow theory is adopted this paper, It means that the gas (vapor)-liquid two-phase fluid is taken as a homogeneous medium. The flow physical parameters are taken from the average corresponding parameters of two-phase medium. Then, the relevant problems are solved in accordance with the fluid dynamics of single-phase medium.

\subsection{Sample solution and results}

The pressure drop from import to the first hole and the pressure drop between each hole can be calculated by straight pipe pressure drop formula of hydraulics. The equations are used as follows:

$$
\begin{gathered}
\Delta P=2 f \rho u^{2} \frac{l}{D} \\
f=\frac{16}{R_{e}} \mathrm{R}_{\mathrm{e}} \leq 2300 \\
f=\frac{0.0791}{\left(R_{e}\right)^{\frac{1}{4}}} \mathrm{R}_{\mathrm{e}}>2300 \\
R_{e}=\frac{\rho D u}{\mu}
\end{gathered}
$$

The rest of the calculation equations are Eq. 7, Eq. 5, Eq. 4 and Eq. $6 .$.

The calculative steps are: (1) Given $P_{0}$, (2) The calculation of $R_{\mathrm{e}}$, (3) $\mathrm{f}$ is calculated according to the value of $\mathrm{R}_{\mathrm{e}}$ to decide whether to use Eq.9 or Eq.10, (4) Apply the Eq.8 to calculate the $\triangle P$, (5) the calculation of $P_{1}, P_{1}=P_{0}+\triangle P$, (6) Apply the Eq.6 to calculate the $a$, (7) Apply the Eq.7、 Eq.5 and Eq.4 to calculate the $P_{2} 、 u_{\mathrm{e}}$ and $u_{2}$, 8)The calculation of the mass flow rate of the holes $W$, $W=\pi / 4 \cdot d^{2} \cdot \rho \cdot u_{\mathrm{e}}$, 9) The calculation of $R_{\mathrm{e}}$ after Replacing the $u_{0}$ with $u_{2}$, and repeat the above steps, until the fourth hole.

If the downstream velocity of the fouth hole $u_{2}$ is not 0 , then, the trial is restarted given an new $\mathrm{P}_{0}$. The computing is finished until $u_{2} \approx 0$.

Through the above calculation, the results of this example are as follows:

The first hole: $P_{1}=145030 \mathrm{~Pa}, W_{1}=1.590 * 10^{-2} \mathrm{~kg} / \mathrm{s}$ 
The second hole: $P_{2}=145060 \mathrm{~Pa}, W_{2}=1.591 * 10^{-2} \mathrm{~kg} / \mathrm{s}$

The third hole: $P_{3}=145083 \mathrm{~Pa}, W_{3}=1.591 * 10^{-2} \mathrm{~kg} / \mathrm{s}$

The fourth hole: $P_{4}=145089 \mathrm{~Pa}, W_{4}=1.591 * 10^{-2} \mathrm{~kg} / \mathrm{s}$

The downstream velocity of the fourth hole in the string $u_{2}=4.80^{*} 10^{-2} \mathrm{~m} / \mathrm{s}, u_{2} \approx 0$. So, the calculation results conform to requirement.

\section{Conclusion}

(1) The axial multipoint steam injection string of the horizontal well, which is designed by the pressure calculation equation in this paper, can ensure an even steam output of each point. It means that the flow quantity of each hole is equal or similar

(2) The results of analysis and calculation show that the theory and calculation method can be used for the design of the multipoint steam injection string of the horizontal well in oil field.

\section{Nomenclature}

$P=$ pressure; $u$ = flow velocity; $\rho$ = density of flow fluid in the string; $\mu=$ viscosity of fluid;

$u_{\mathrm{e}}=$ exit flow velocity at side holes of steam injection string; $D=$ diameter of string;

$C=$ orifice metering coefficient, determined by experiment; $f=$ Fanning friction factor

\section{References}

[1] Novy R A. Pressure drops in horizontal wells: when can they be ignored [J]. SPE 24941,1992.

[2] Wang Qing, Liu Huiqing, Zhang Hongling, Zheng Jiapeng. An optimization model of completion strings with inner-located nozzle in horizontal wells coupled with reservoirs[P]GB.2007200113343.7,2008-4-30. 